\title{
An ultrafast reconfigurable nanophotonic switch using wavefront shaping of light in a nonlinear nanomaterial
}

\author{
Tom Strudley ${ }^{1}$, Roman Bruck ${ }^{1}$, Ben Mills ${ }^{2}$ and Otto L Muskens ${ }^{1}$ \\ We demonstrate a new concept for reconfigurable nanophotonic devices exploiting ultrafast nonlinear control of shaped wavefronts in a \\ multimode nanomaterial consisting of semiconductor nanowires. Femtosecond pulsed laser excitation of the nanowire mat is shown to \\ provide an efficient nonlinear mechanism to control both destructive and constructive interference in a shaped wavefront. Modulations \\ of up to $63 \%$ are induced by optical pumping, due to a combination of multimode dephasing and induced transient absorption. We \\ show that part of the nonlinear phase dynamics can be inverted to provide a dynamical revival of the wavefront into an optimized spot \\ with up to $18 \%$ increase of the peak to background ratio caused by pulsed laser excitation. The concepts of multimode nonlinear \\ switching demonstrated here are generally extendable to other photonic and plasmonic systems and enable new avenues for ultrafast \\ and reconfigurable nanophotonic devices.
}

Light: Science \& Applications (2014) 3, e207; doi:10.1038/lsa.2014.88; published online 26 September 2014

Keywords: nanophotonics; nanowires; nonlinear optics; reconfigurable; ultrafast; wavefront shaping

\section{INTRODUCTION}

Many of the available photonic technologies are based on perfectly regular, ordered structures such as waveguides, photonic crystals and metamaterials. There is, however, an increasing interest to exploit the additional degrees of freedom offered by aperiodic or disordered designs. ${ }^{1-4}$ One way of controlling the flow of coherent energy transfer in such a medium with high efficiency is through optimization of the specific arrangement of the scatterers. ${ }^{5,6}$ Exciting new techniques have emerged based on shaping of the light field itself to match a given scattering configuration, either through time reversal ${ }^{7,8}$ or iterative schemes. ${ }^{9,10}$ The method of wavefront shaping is based on the general concept that the transmission through any medium can be described by a matrix which connects all ingoing and outgoing degrees of freedom. In principle, knowledge of the transmission matrix, ${ }^{11,12}$ along with an ability to completely control the incident light, ${ }^{10}$ would allow the selection of any desired output, turning an opaque medium into a versatile optical element. Next to the interest for biomedical imaging, ${ }^{13-15}$ wavefront shaping shows promise for reconfigurable optical elements ${ }^{16-20}$ and control of random lasers. ${ }^{21}$ While initial work concentrated on monochromatic continuous-wave radiation, focusing through opaque scattering media has also been achieved using ultrashort pulses ${ }^{22,23}$ and polychromatic light. ${ }^{24}$

Here, we demonstrate both destructive and constructive switching of a shaped wavefront on ultrafast time scales through nonlinear optical excitation of the scattering medium. With the rapid development of applications exploiting wavefront shaping, active control of such shaped fields is of great interest. The principle is illustrated in Figure 1a. Wavefront shaping amounts to aligning the phasors resulting from independent light paths in the medium to produce a predefined output pattern, such as a single sharp focus. ${ }^{10}$ The optimized state relies on coherence between modes exploring completely different trajectories in space and time, which can be easily perturbed by small changes to the medium. Femtosecond optical excitation of a semiconductor produces a series of nonlinear phase shifts (denoted by $\Delta \phi$ in Figure 1a) which give rise to uncorrelated, but reproducible changes in the transmission mode spectrum, as was demonstrated in our previous work. ${ }^{25}$ In those earlier studies, the output of the medium was a random speckle pattern, limiting its use for applications. In the current work, we extend the idea of multimode dephasing by combining it with wavefront shaping to control both the destructive and constructive interference in a shaped light field on ultrafast time scale. By harnessing the output through constructive wavefront shaping, the nonlinear effects become useful and can be applied in realistic devices. Moreover, we proceed beyond dephasing by demonstrating that the dynamics can be reversed to provide a revival of a shaped wavefront on ultrafast time scales. The fact that pump-induced, nonlinear phase dynamics can be used to increase the constructive interference of a coherent superposition state was not anticipated from Ref. 25 and opens up new prospects for coherent control and wavefront shaping of light in nonlinear media.

\section{MATERIALS AND METHODS}

For our investigations, we make use of mats of GaP nanowires. While previously we have used such samples for investigations of fundamental mesoscopic physics of light, ${ }^{26}$ it is the nonlinear response of such nanowires that is of interest to this study. ${ }^{25}$ The optical thickness of these $4.5-\mu \mathrm{m}$ thick mats is more than 20 optical mean free paths,

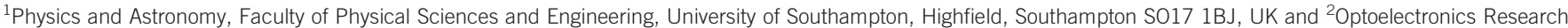
Centre, Faculty of Physical Sciences and Engineering, University of Southampton, Highfield, Southampton SO17 1BJ, UK

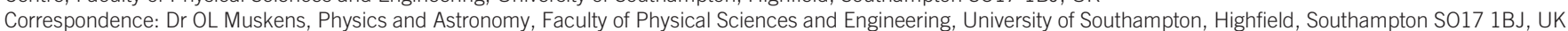
E-mail: o.muskens@soton.ac.uk

Received 4 May 2014; revised 15 July 2014; accepted 17 July 2014 
which is sufficient to suppress any coherent beam transmission and to produce a complete mixing of input and output degrees of freedom. ${ }^{26,27}$

The set-up, as shown in Figure 1b, combines a wavefront shaping arrangement with two-color, femtosecond pump-probe spectroscopy. A regenerative amplifier (Coherent RegA, Coherent, Santa Clara, CA, USA. $250 \mathrm{kHz}, 150 \pm 10$ fs pulse duration) pumped optical parametric amplifier (Coherent OPA 9400, Coherent, Santa Clara, CA, USA) was used to provide probe and pump light with center wavelengths of $633 \mathrm{~nm}$ and $400 \mathrm{~nm}$, respectively. A 1-nm narrowband spectral filter was used to reduce the bandwidth of the probe pulses to $0.75 \mathrm{THz}$, close to the characteristic frequency correlation width of the speckle in the medium. ${ }^{25,26}$ This allowed us to perform wavefront shaping in the spatial domain using only a single frequency mode (as opposed to temporal shaping $\left.{ }^{22,23}\right)$. As a consequence of the spectral filtering, the pulse duration of the probe was increased to 5 ps.

The pump and probe were focused onto the nanowires using a microscope objective (Nikon CFI60 LU Plan BD, Nikon instruments, Kingston upon Thames, UK) with numerical aperture of 0.9. By reducing the pump collimation before the objective (L6 in Figure 1b), we achieved a homogeneous pump illumination over the entire $20-\mu \mathrm{m}$ diameter area used for wavefront shaping. The total average incident power of the pump on the sample was $8 \mathrm{~mW}$, while the used probe power was less than $0.1 \mathrm{~mW}$. The pump fluence on the sample was estimated to be $15 \mathrm{~mJ} \mathrm{~cm}^{-2}$, approximately $70 \%$ of the threshold at which slow, photo-induced sample degradation was observed. The time delay between probe and pump pulses was controlled using a mechanical stage, and was calibrated using the nonlinear response of a bare GaP slab without any scatterers. ${ }^{25,28}$ For each delay position, an image was taken with and without pump using a computer controlled shutter to block the pump beam in order to obtain the relative effect of the pump. Transmitted light was collected by a second, identical objective and imaged onto a monochrome 16-bit camera (AVT Stingray, Allied Vision Technologies, Stadtroda, Germany).

To produce an optimized spot, a digital micromirror device (DMD; Vialux, Chemnitz, Germany) was used to control the incident probe light. We used a binary amplitude modulation scheme by Akbulut et $a .^{29}$ to switch off transmission modes that interfere destructively at a selected point, leaving only modes that interfere constructively to create a single bright focus. In the experiment, the DMD was divided into segments of 20 pixels $\times 20$ pixels, providing a total of 900 independently controlled incident modes. The time to optimize a single segment was $30 \mathrm{~ms}$, resulting in a total optimization time of $30 \mathrm{~s}$. A further optimization cycle was then performed to benefit from the improved signal-to-noise ratio in the pre-optimized spot, after which no significant improvement was obtained for further cycles with a stable configuration. By positioning the focal plane of the collection objective approximately $5 \mu \mathrm{m}$ behind the exit surface, optimizations were performed on a single spot in the far field of the scattering slab.

Images were processed using Matlab (Mathworks, Natick, MA, USA). For each data point, areas of interest were selected from the images corresponding to either the peak or background region. Integrated intensities for images with pump were normalized to values without pump to obtain normalized data for each time delay.

\section{RESULTS AND DISCUSSION}

\section{Dephasing of a shaped wavefront}

Figure 2a shows the optimized spot obtained using the picosecond probe pulse, with a radially averaged cross-section shown in Figure 2c (black line). An order of magnitude enhancement was obtained, which is limited by the amplitude modulation scheme combined with the finite laser bandwidth and intrinsic noise of the parametric amplifier. Ultimately, the maximum possible enhancement is set by the available number of independent transmission channels, ${ }^{30}$ which is around 50 for the sample and illumination conditions under study. ${ }^{26}$

After optimization of the wavefront, the pump illumination was switched on in order to produce a nonlinear modulation of the shaped light field. Figure $2 \mathrm{~b}$ shows the intensity map for the maximum of the pump-probe dephasing effect at 4 ps delay time, with the cross-section shown in Figure 2c (red line). Figure 2d shows the pump-probe time dynamics of the transmitted intensity $T_{\text {pump }}$ normalized to its value without pump $T_{\text {nopump }}$, for the optimized spot (black line) and the average background intensity (red line). The peak to background ratio (blue line) is defined as the ratio between optimized spot and background. The main effect is a reduction of the intensity in the optimized spot down to $37 \%$ of the value without pump, corresponding to a modulation contrast of $4.3 \mathrm{~dB}$. This reduction of the shaped spot is much stronger than that of the background intensity, which is reduced by only half this amount. In addition to the ultrafast effect,
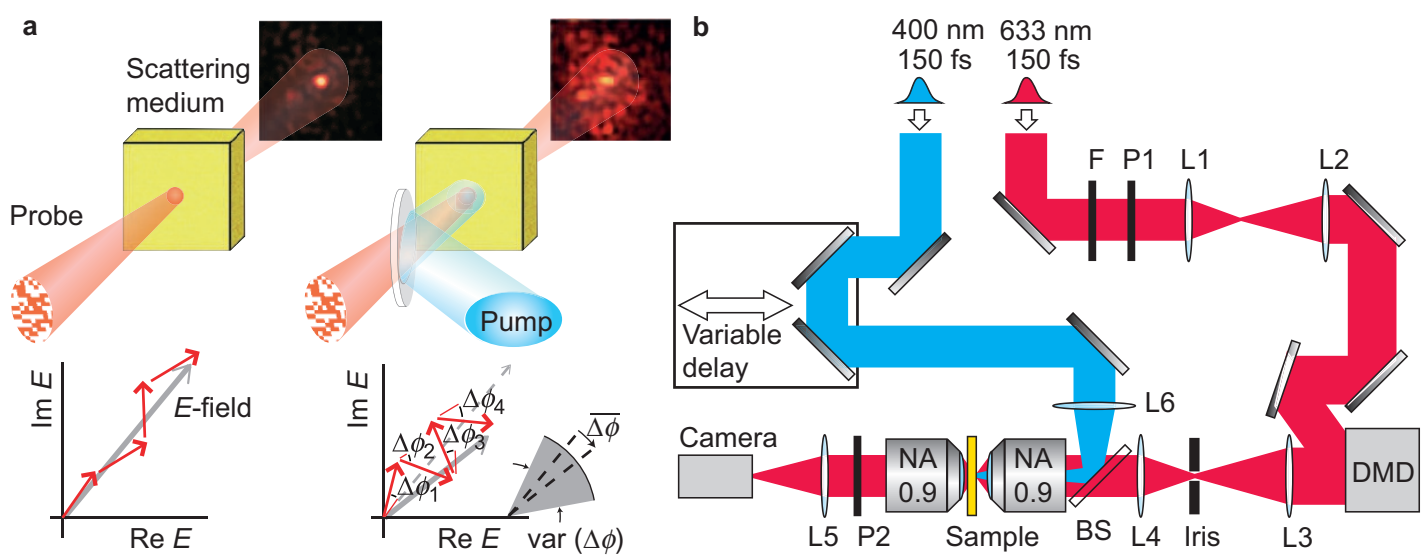

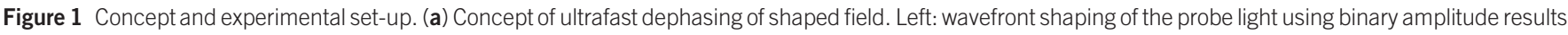

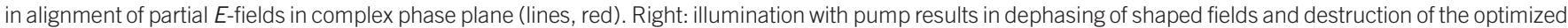

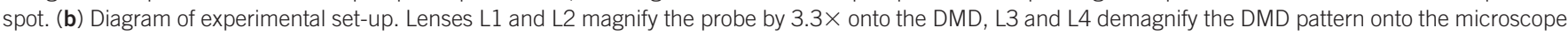

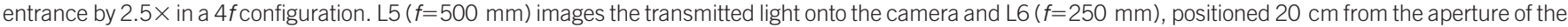

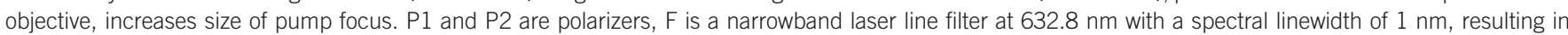
broadening of the probe pulse duration to $5 \mathrm{ps}$. DMD, digital micromirror device; NA, numerical aperture. 

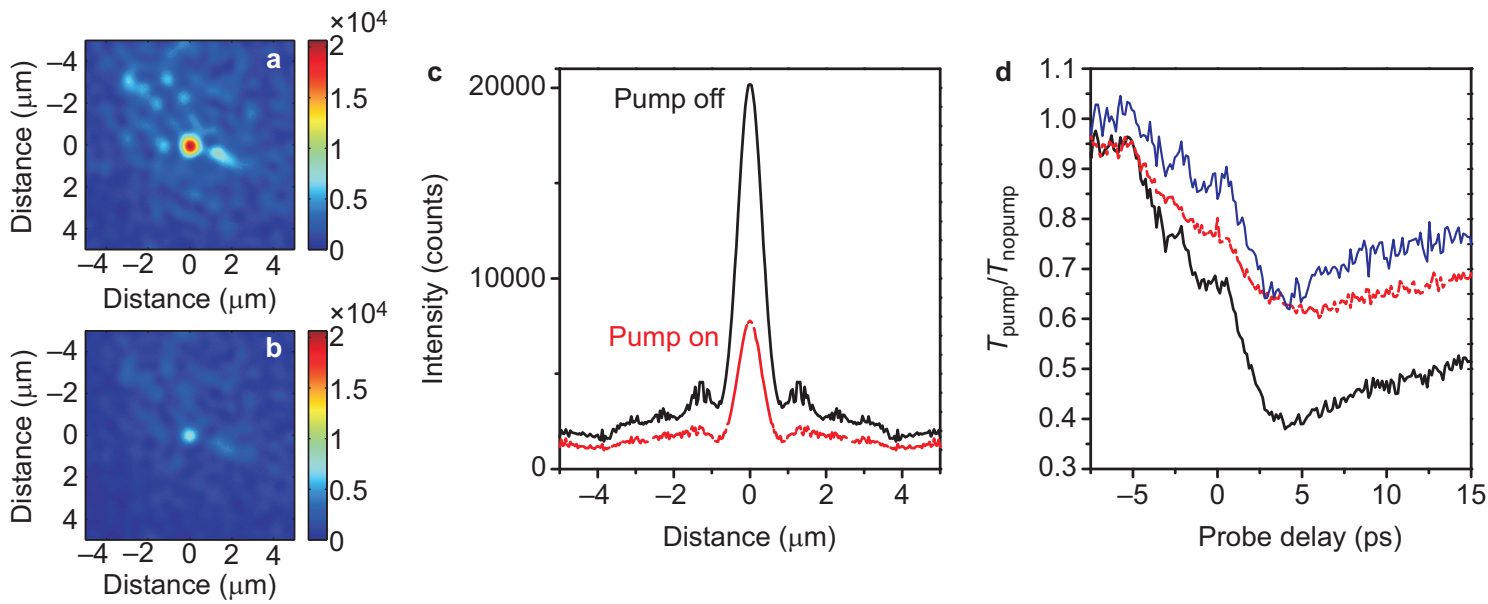

Figure 2 Nonlinear control of optimized spot. Images of a shaped wavefront optimized for pump off (a) and pump on (b) conditions. (c) Radially averaged crosssections of $\mathbf{a}, \mathbf{b}$ for pump off (line, black) and pump on (dashed line, red) conditions. The change in peak intensity is $63 \%$ with a pump delay of 2 ps. (d) Plots of the transmission in the presence of the pump $T_{\text {pump }}$, normalized to value without pump $T_{\text {nopump }}$, as a function of pump delay for the peak intensity of the optimized spot (thick line, black), and the average background intensity (dashed line, red). Thin line, blue denotes peak to background ratio.

both the peak and the background intensities show a $T_{\text {pump }} / T_{\text {nopump }}$ ratio of around $95 \%$ for delay times less than $-5 \mathrm{ps}$, due to a pumpinduced heat pileup in the nanowires.

The nonlinear switching effect was found to be reproducible for different positions on the sample corresponding to different multiple scat-
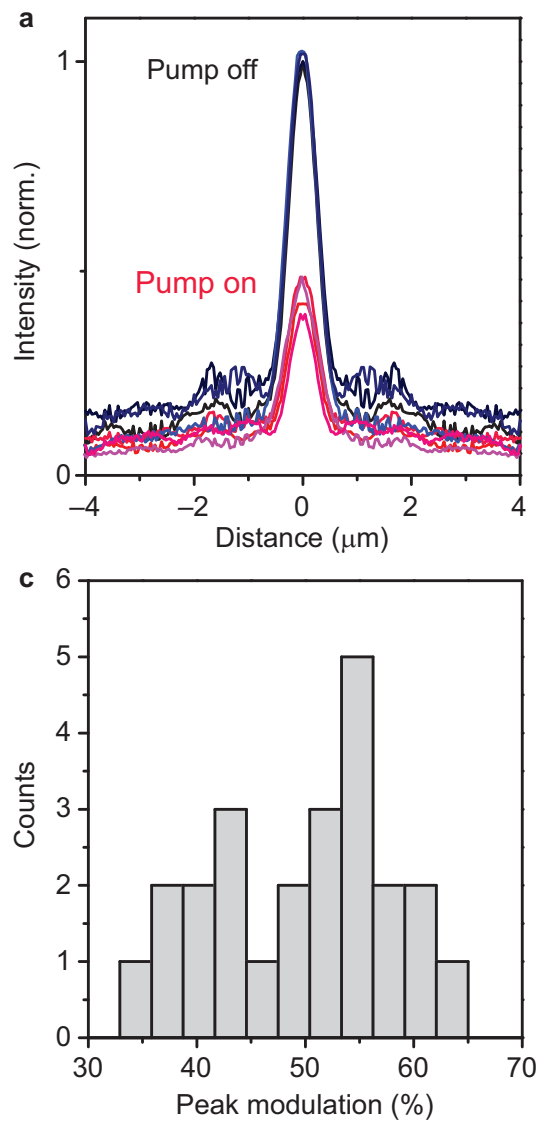

tering configurations. Figure 3a shows the normalized peak modulation for three different sample positions, while statistical results for 24 different positions are given in Figure $3 \mathrm{~b}-3 \mathrm{~d}$. The peak to background enhancement using the binary optimization scheme varied between 8 and 16 for these optimizations (Figure $3 \mathrm{~b}$ ). The mean value of the
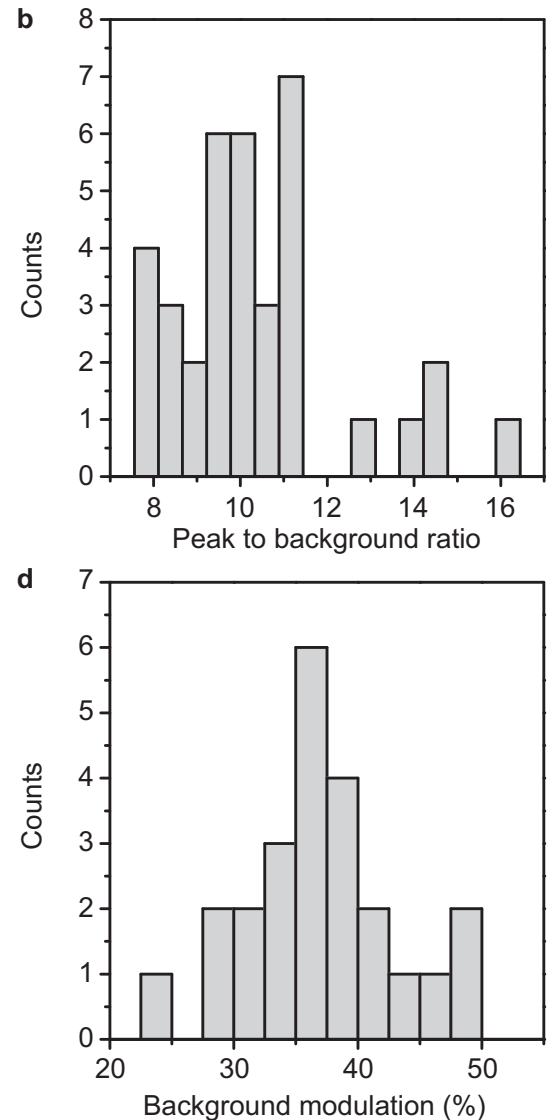

Figure 3 Reproducibility of nonlinear switching effect. (a) Radially averaged profiles of optimized spot without pump on three different sample positions (black-blue lines, pump off) and in the presence of the pump (red-magenta lines, pump on). Histograms demonstrating the variation in experimental results for switching a spot optimized without pump, for maximum peak optimization (b), modulation of the peak intensity (c) and integrated background away from peak (d). 
modulation of the peak achieved at different sample positions was $(50 \pm 2) \%$ (Figure $3 \mathrm{c}$ ), while the modulation of the background was found to be $(36 \pm 2) \%$ (Figure $3 \mathrm{~d}$ ). In addition to variations in magnitude of the switching effect, the detailed dynamics of the optimized spot were found to also depend sensitively on the local scattering configuration, as is shown in Figure 4a for three representative positions corresponding to those of Figure $3 \mathrm{a}$. The variation in dynamics of the peak (labeled A-C in Figure 4a) reflects the limited number of independent transmission modes in the optimized spot. ${ }^{30}$ As each mode is associated with a specific set of light paths through the medium, the time evolution represents the characteristic dwell times of the subset of modes that are contributing to the shaped light field for each particular configuration. Compared to the optimized spot, the integrated background intensity consists of a much larger number of transmission modes, resulting in a much smoother behavior which did not depend markedly on sample positions (red lines in Figure 4a).

In the absence of mesoscopic effects, the modulation of the background intensity can be described by pump-induced transient absorption during the diffuse transport time of light through the nanowire mat. $^{25,31}$ If there was only absorption, the optimized peak should follow the background and no modulation of the peak to background ratio would be observed. From the peak to background ratio, we conclude that the effect of absorption accounts for less than half of the total switching of the peak, and the remainder reflects the effect of pure dephasing processes in the switching. Further evidence of the presence of ultrafast dephasing effects is obtained by looking at the phase coherence of the background speckle. Dephasing effects result in a redistribution of intensity over the different transmission modes, ${ }^{25}$ which is not visible when looking only at the integrated transmission intensity. Therefore, we calculated the cross-correlation $C$ of the spatial intensity maps taken with ('p') and without pump ('np') at each delay time, given by

$$
C_{\mathrm{p}, \mathrm{np}}=\frac{\sum_{x, y}\left[I_{\mathrm{p}}(x, y)-\left\langle I_{\mathrm{p}}\right\rangle\right]\left[I_{\mathrm{np}}(x, y)-\left\langle I_{\mathrm{np}}\right\rangle\right]}{\sqrt{\sum_{x, y}\left[I_{\mathrm{p}}(x, y)-\left\langle I_{\mathrm{p}}\right\rangle\right]^{2}} \sqrt{\sum_{x, y}\left[I_{\mathrm{np}}(x, y)-\left\langle I_{\mathrm{np}}\right\rangle\right]^{2}}}
$$

where brackets denote the average value over the selected area of interest of the image. We find a decorrelation of the speckle pattern in the presence of pump illumination as shown in Figure $4 \mathrm{~b}$ for the three positions. The time dependence of this decorrelation matches well the dynamics of the corresponding peaks $\mathrm{A}-\mathrm{C}$ for each configuration, evidencing the strong role of dephasing in the modulation of the optimized wavefront.

\section{Ultrafast revival of a shaped wavefront}

The above results show that it is possible to selectively destroy the constructive interference of a shaped light field using the nonlinearity of the scattering medium. Our finding that nearly half of the switching is caused by pure dephasing leads to the exciting question whether the phase dynamics can be inverted to achieve constructive interference in the presence of the pump. We tested the potential of such a dynamical rephasing effect in an experiment where the probe field was shaped in the presence of pump illumination and for a given pump-probe delay. Figure $5 \mathrm{a}$ and $5 \mathrm{~b}$ summarizes the results obtained for a range of optimizations at different pump-probe delay times. Here, the vertical black arrows represent the pump-probe delay time at which the optimization was done (the horizontal dashed lines are the baselines of the vertically shifted curves). The first thing to notice is that the background intensity (Figure 5a) is not affected at all by the optimization. This is to be expected since the background modulation only depends on transient absorption.

In comparison, the peak to background ratio (Figure 5b) shows pronounced differences between optimization with and without pump. For optimization without pump, we see for this particular configuration the suppression of the shaped field beginning around zero time delay with a maximum effect of $(20 \pm 2) \%$ at 3.5 ps.

For optimization with pump on, we find that the dynamics of the peak to background ratio strongly depend on the exact timing of the pump and probe pulses used during the optimization process. For a range of optimization delay times between -1.5 ps and $5.5 \mathrm{ps}$, the shaped field shows a clear enhancement with pump compared to without pump around the time where the optimization was done. The largest rephasing effect of $(18 \pm 2) \%$ is found for the optimization with a probe delay of $2.2 \mathrm{ps}$, close to the delay at which the largest dephasing effect occurs for the optimization with pump off. Figure $5 \mathrm{~d}$ and $5 \mathrm{e}$ shows a more detailed analysis of the dynamics taken at the optimization at 2.2 ps probe delay. The increase of the pump to background ratio is most clearly observed in Figure 5e. This increase cannot be explained by an absorption difference between peak and background, as this would not show a maximum which shifts with the optimization time delay. We therefore attribute the relative increase of the shaped field to a coherent rephasing, driven by the pump-induced changes of the scattering medium.
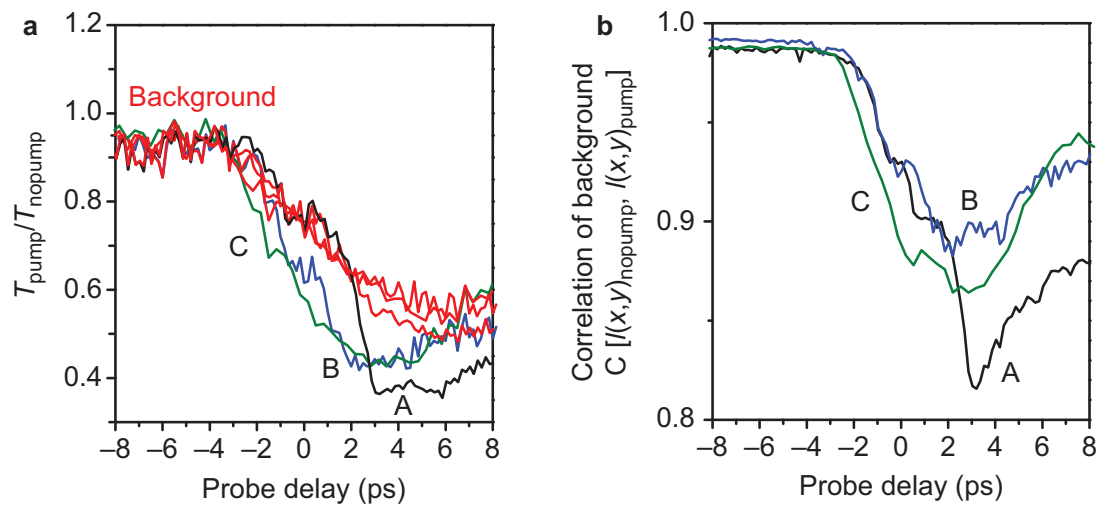

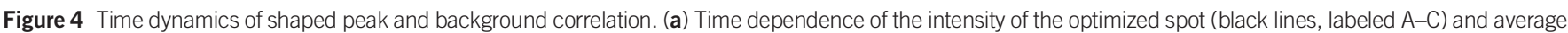

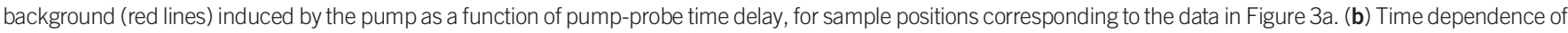

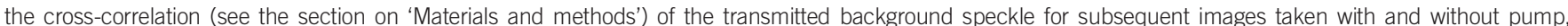
corresponding to sample positions A-C. 

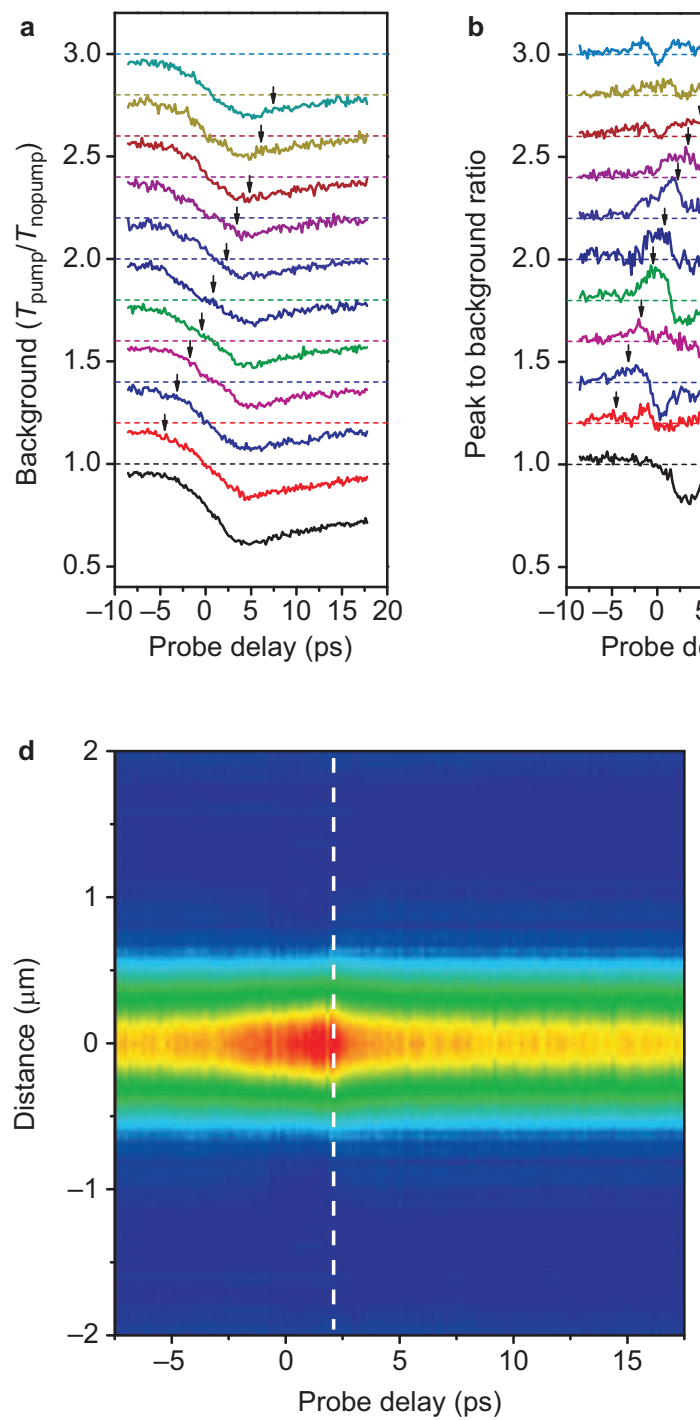
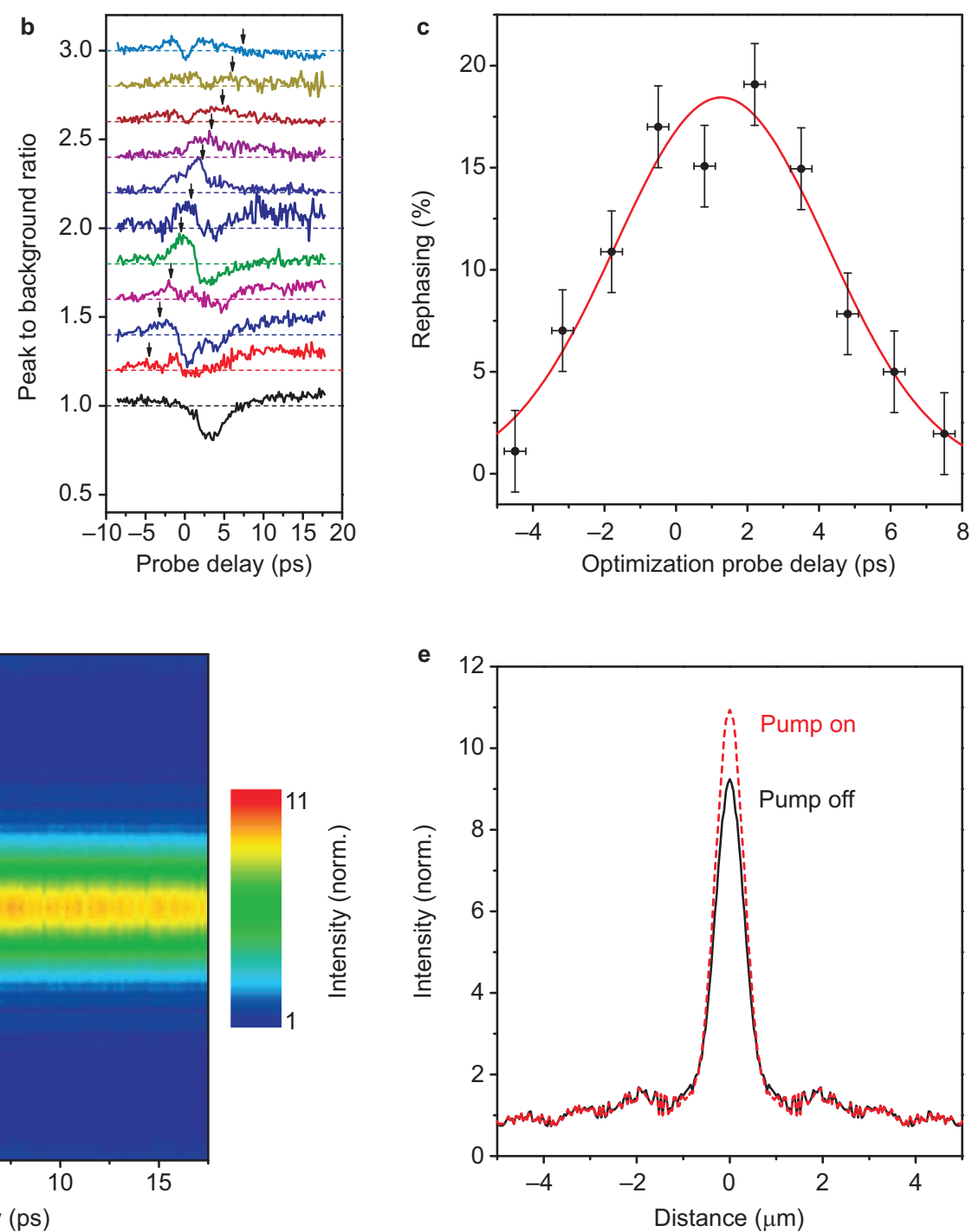

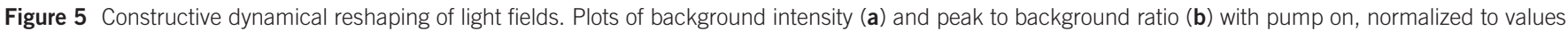

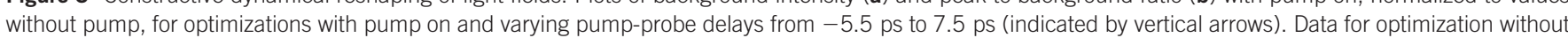

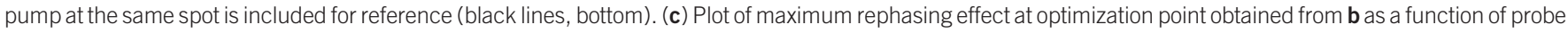

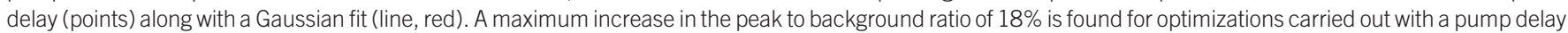

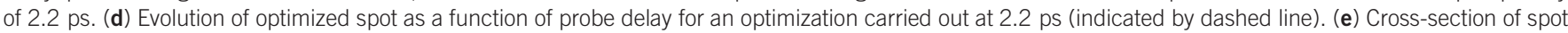

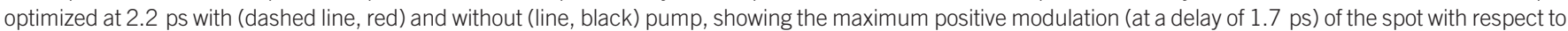
the background by the pump. Both $\mathbf{d}$ and $\mathbf{e}$ were radially averaged and normalized to the average background.

We reproduced the dynamic rephasing effect on several areas of the sample as shown in Figure 6. Figure 6a shows the intensity crosssections at the maximum rephasing time delay, normalized to the background intensity at $4 \mu \mathrm{m}$ distance from the peak, for three independent sample positions. The full time dynamics of the rephasing effect for these different configurations are shown in Figure 6b-6d. The arrows indicate the time delay at which the optimization was done in the presence of pump illumination. While nominally the same amount of maximum rephasing is observed in the peak to background ratio (blue lines), there are again variations in the dynamics depending sensitively on the sample position. As the nonlinearity will impact differently on each transmission mode, the specific dynamics in this region are likely to be an individual fingerprint of the scattering configuration. This behavior is in agreement with that observed for the dephasing effect, and shows that both the linear transmission and the nonlinear phase dynamics can potentially be engineered by a rational design of the scattering configuration.

Our results show that a large fraction of the nonlinear dephasing can be inverted to produce a coherent reshaping of the wavefront. Some losses in the inversion could be caused by nondeterministic or inelastic dephasing processes. We expect that in particular the effects of nonadiabatic modulation processes occurring on a time scale faster than the dwell time, ${ }^{32,33}$ or reciprocity breaking ${ }^{28}$ will produce phase dynamics and nonlinear spectral broadening which cannot effectively be harnessed for rephasing of the entire probe pulse.

\section{Comparison with other switching concepts}

Optical switching through multimode dephasing in a complex medium is a conceptually novel approach to all-optical control. Other semiconductor devices showing ultrafast phase modulation include 

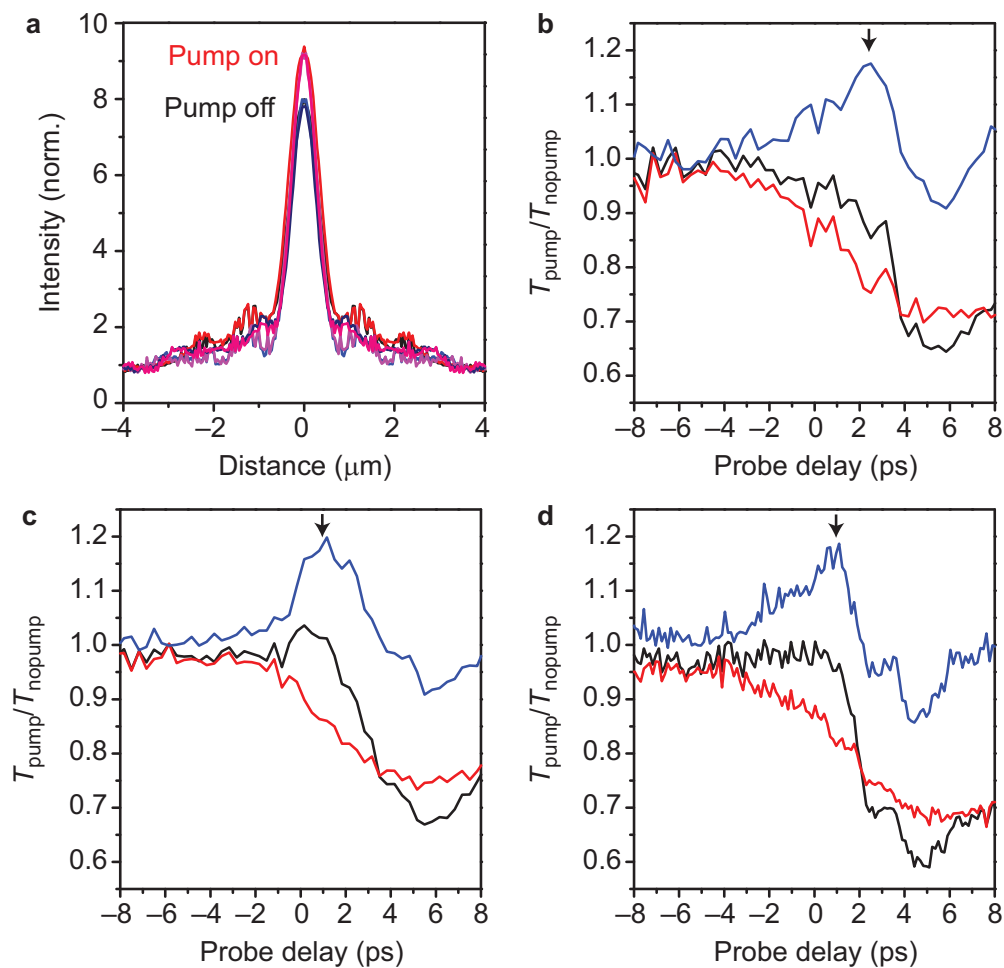

Figure 6 Reproducibility of constructive dynamical reshaping. (a) Radially averaged cross-sections of peak optimized in the presence of pump for conditions with pump (red magenta lines) and without pump (black-blue lines). (b-d) Time-dependence of the intensity of the optimized spot (black lines) and average background (red lines) for three different sample positions and for optimization with pump on at time delay given by arrows. Blue lines represent peak to background ratio, showing a dynamic reshaping of around $20 \%$ for all positions.

photonic crystals, ring resonators and microcavities. ${ }^{33-40}$ In these devices, typically a single resonant mode is modulated through ultrafast changes in the refractive index, resulting in a deterministic frequency shift of this mode. On the other hand, a Mach-Zehnder interferometer (MZI) is based on the phase coherence between two spatially separate pathways. Our approach can be interpreted as a multipath extension of the MZI in a highly compact configuration where many light paths are folded into a single slab of material. The spatiotemporal multimode complexity of the different paths showing different phase dynamics results in a complete reshaping of the mode spectrum. Thus, rather than a small frequency shift, we see a complete collapse-or revival_of the output mode. Our device concept thus provides a switching characteristic which is very different from any other nonlinear switching devices.

While differences in device geometries render a direct comparison between one-, two- and three-dimensional structures difficult, a simple but instructive approximation is to look at the characteristic nonlinear interaction times. The accumulated nonlinear phase $\Delta \phi$ is proportional to the time $\tau$ the light spends in the excited region according to $\Delta \phi=\Delta n \omega \tau$, with $\Delta n$ the nonlinear refractive index change and $\omega$ the angular frequency. For an MZI of arm length $L$, the interaction time is $\tau_{\mathrm{MZI}}=L / c$, whereas for a cavity, this time is increased to $\tau_{\mathrm{C}}=F L / \pi c$, with $F$ the cavity finesse, $L$ the cavity length and $c$ the speed of light. For a complex scattering medium, the average time light takes to diffuse through the excited slab of thickness $L$ is the Thouless time $\tau_{\mathrm{D}}=3 L^{2} / c l$, where $l$ is the transport mean free path of light in the scattering medium. ${ }^{41}$ For equal length $L$ of the excited region, the scattering medium thus provides the same accumulated phase as a cavity with finesse $F=3 \pi L / l$, amounting to around 200 for our studies. Indeed, earlier experiments ${ }^{25}$ showed a frequency shift of the overall speckle pattern of our random medium corresponding to the average accumulated nonlinear phase $\overline{\Delta \phi}$ as illustrated in Figure 1a. In comparison, a much longer device length (60 times in our example) is required for the MZI to obtain the same accumulated nonlinear phase.

In addition to the small average frequency shift, a much larger effect in our samples is caused by pure dephasing of modes. ${ }^{25}$ Dephasing results in the decorrelation of intensity induced by a random distribution of accumulated phases proportional to $C_{\mathrm{p}, \mathrm{np}} \approx\left\langle e^{i \Delta \phi}\right\rangle \equiv e^{-1 / 2\left\langle\Delta \phi^{2}\right\rangle}$ where the equivalence assumes a Gaussian distribution and the brackets denote an average over the ensemble of modes contributing to the intensity. ${ }^{41}$ The decoherence model predicts a $1 / e$ reduction of the intensity for a Gaussian distribution of accumulated phases of width $\operatorname{var}(\Delta \phi)=2$. The dephasing mechanism thus benefits from a broad distribution of path lengths, which is provided by the power-law distribution of diffuse transport times through the slab, ${ }^{41}$ as well as from spatiotemporal inhomogeneity of the excitation zone. The latter condition is fulfilled by the fast time dynamics, limited penetration depth and intrinsic intensity fluctuations of pump light inside the sample, but could potentially be further optimized by structuring of the pump illumination to imprint specific nonlinear potential landscapes. ${ }^{42}$

\section{Future prospects}

While the efforts in our current work mainly focused on the proof of concept of ultrafast switching, much higher efficiencies and speeds of wavefront shaping can be easily implemented. ${ }^{9-15}$ The use of full phase control in wavefront shaping will result in more significant enhancement in the peak to background ratios achieved. Moreover, knowledge 
of the transmission matrix of the material will allow generating any predetermined configuration on command within the switching time of the DMD, i.e., at $\mathrm{kHz}$ speeds. ${ }^{11}$ Such speeds are of interest for a variety of applications in displays and imaging. Ultimately, we envisage that multimode devices could form the nodes of an adaptive network, where the multimode complexity provides the reconfigurability and picosecond control performs the function of all-optical communication gate. Particularly exciting is the prospect of implementing these general concepts in two dimensional plasmonic and nanophotonic waveguides, which could result in a new paradigm for reconfigurable networks.

Another application range which could take profit from the picosecond switching speed is that of ultrafast shutters for time-resolved spectroscopy. High-throughput devices may be obtained by designing configurations that match the open transmission eigenchannels of the medium with order unity transmission. ${ }^{10,12}$ A rational design of arrangements with predefined characteristics ${ }^{6}$ will allow engineering of both the linear energy transfer and nonlinear phase dynamics similar to complex molecular systems. ${ }^{43}$

\section{CONCLUSIONS}

In conclusion, we have demonstrated ultrafast optical modulation of a shaped light field by up to $63 \%(4.3 \mathrm{~dB})$, with both dephasing and absorption performing important roles. The effect of dephasing can be partially inverted to produce a constructive revival of the light field in the presence of a femtosecond pulsed excitation. The maximum positive modulation found using this striking rephasing effect is $18 \%$. The presence of a nonlinear scattering medium therefore adds new ways to control the transmitted light, on top of the already impressive possibilities offered by wavefront shaping.

\section{ACKNOWLEDGMENTS}

The authors thank E Bakkers from Eindhoven University of Technology for providing the nanowire sample. OLM and RB acknowledge financial support from EPSRC through grant EP/J016918/1.

1 Maciá E. Exploiting aperiodic designs in nanophotonic devices. Rep Prog Phys 2012 75: 036502 .

2 Dal Negro L, Boriskina SV. Deterministic aperiodic nanostructures for photonics and plasmonics applications. Laser Photon Rev 2012; 6: 178-218.

3 Wiersma DS. Disordered photonics. Nat Photonics 2013; 7: 188-196.

4 Levi L, Rechtsman M, Freedman B, Schwartz T, Manela 0 et al. Disorder-enhanced transport in photonic quasicrystals. Science 2011; 332: 1541-1544.

5 Tanemura T, Balram KC, Ly-Gagnon DS, Wahl P, White JS et al. Multiple-wavelength focusing of surface plasmons with a nonperiodic nanoslit coupler. Nano Lett 2011; 11: 2693-2698.

6 Liu V, Jiao Y, Miller DA, Fan S. Design methodology for compact photonic-crystalbased wavelength division multiplexers. Opt Lett 2011; 36: 591-593.

7 Lerosey G, de Rosny J, Tourin A, Fink M. Focusing beyond the diffraction limit with farfield time reversal. Science 2007; 315: 1120-1122.

8 Judkewitz B, Wang YM, Horstmeyer R, Mathy A, Yang C. Speckle-scale focusing in the diffusive regime with time reversal of variance-encoded light (TROVE). Nat Photonics 2013; 7: 300-305

9 Vellekoop IM, Mosk AP. Focusing coherent light through opaque strongly scattering media. Opt Lett 2007; 32: 2309-2311.

10 Mosk AP, Lagendijk A, Lerosey G, Fink M. Controlling waves in space and time for imaging and focusing in complex media. Nat Photonics 2012; 6: 283-292.

11 Popoff SM, Lerosey G, Carminati R, Fink M, Boccara AC et al. Measuring the transmission matrix in optics: an approach to the study and control of light propagation in disordered media. Phys Rev Lett 2010; 104: 100601.

12 Kim M, Choi Y, Yoon C, Choi W, Kim J et al. Maximal energy transport through disordered media with the implementation of transmission eigenchannels. Nat Photonics 2012; 6: 581-585.
13 Papagiakoumou E, Bègue A, Leshem B, Schwartz O, Stell BM et al. Functional patterned multiphoton excitation deep inside scattering tissue. Nat Photonics 2013; 7: 274-278.

14 Conkey DB, Caravaca-Aguirre AM, Piestun R. High-speed scattering medium characterization with application to focusing light through turbid media. Opt Express 2012; 20: 1733-1740.

15 Chaigne T, Katz O, Boccara AC, Fink M, Bossy E et al. Controlling light in scattering media noninvasively using the photoacoustic transmission matrix. Nat Photonics 2014; 8: 58-64.

16 Čižmár T, Dholakia K. Exploiting multimode waveguides for pure fibre-based imaging. Nat Commun 2012; 3: 1027.

17 Gjonaj B, Aulbach J, Johnson PM, Mosk AP, Kuipers L et al. Active spatial control of plasmonic fields. Nat Photonics 2011; 5: 360-363.

18 Park JH, Park C, Yu H, Cho YH, Park Y. Dynamic active wave plate using random nanoparticles. Opt Express 2012; 20: 17010-17016.

19 Redding B, Cao H. Using a multimode fiber as a high-resolution, low-loss spectrometer. Opt Lett 2012; 37: 3384-3386.

20 Small E, Katz O, Guan Y, Silberberg Y. Spectral control of broadband light through random media by wavefront shaping. Opt Lett 2012; 37: 3429-3431.

21 Bachelard N, Gigan S, Noblin X, Sebbah P. Adaptive pumping for spectral control of random lasers. Nat Phys 2014; 10: 426-431.

22 Aulbach J, Gjonaj B, Johnson PM, Mosk AP, Lagendijk A. Control of light transmission through opaque scattering media in space and time. Phys Rev Lett 2011; 106: 103901.

23 Katz O, Small E, Bromberg Y, Silberberg Y. Focusing and compression of ultrashort pulses through scattering media. Nat Photonics 2011; 5: 372-377.

24 Paudel HP, Stockbridge C, Mertz J, Bifano T. Focusing polychromatic light through strongly scattering media. Opt Express 2013; 21: 17299-17308.

25 Abb M, Bakkers EP, Muskens OL. Ultrafast dephasing of light in strongly scattering GaP nanowires. Phys Rev Lett 2011; 106: 143902.

26 Strudley T, Zehender T, Blejean C, Bakkers EP, Muskens OL. Mesoscopic light transport by very strong collective multiple scattering in nanowire mats. Nat Photonics 2013; 7: 413-418.

27 Muskens OL, Diedenhofen SL, Kaas BC, Algra RE, Bakkers EP et al. Large photonic strength of highly tunable resonant nanowire materials. Nano Lett 2009; 9: 930-934.

28 Muskens OL, Venn P, van der Beek T, Wellens T. Partial nonlinear reciprocity breaking through ultrafast dynamics in a random photonic medium. Phys Rev Lett 2012; 108 : 223906.

29 Akbulut D, Huisman TJ, van Putten EG, Vos WL, Mosk AP. Focusing light through random photonic media by binary amplitude modulation. Opt Express $2011 ; 19$. 4017-4029.

30 Davy M, Shi Z, Genack AZ. Focusing through random media: eigenchannel participation number and intensity correlation. Phys Rev B 2012; 85: 035105.

31 Versteegh MA, van der Wel RE, Dijkhuis JI. Measurement of light diffusion in ZnO nanowire forests. App/ Phys Lett 2012; 100: 101108.

32 Yacomotti AM, Raineri F, Cojocaru C, Monnier P, Levenson JA et al. Nonadiabatic dynamics of the electromagnetic field and charge carriers in high- $Q$ photonic crystal resonators. Phys Rev Lett 2006; 96: 093901.

33 Notomi M. Manipulating light with strongly modulated photonic crystals. Rep Prog Phys 2010; 73: 096501.

34 Jin CY, Wada O. Photonic switching devices based on semiconductor nano-structures. J Phys D Appl Phys 2014; 47: 133001.

35 Almeida VR, Barrios CA, Panepucci RR, Lipson M. All-optical control of light on a silicon chip. Nature 2004; 431: 1081-1084.

36 Yüce E, Ctistis G, Claudon J, Dupuy E, Buijs RD et al. All-optical switching of a microcavity repeated at terahertz rates. Opt Lett 2013; 38: 374-376.

37 Nozaki K, Tanabe T, Shinya A, Matsuo S, Sato T et al. Sub-femtojoule all-optical switching using a photonic-crystal nanocavity. Nat Photonics 2010; 4: 477-483.

38 Fushman I, Waks E, Englund D, Stoltz N, Petroff P et al. Ultrafast nonlinear optical tuning of photonic crystal cavities. Appl Phys Lett 2007; 90: 091118.

39 Tan HW, van Driel HM, Schweizer SL, Wehrspohn RB, Gösele U. Nonlinear optica tuning of a two-dimensional silicon photonic crystal. Phys Rev B 2004; 70: 205110.

40 Reed GT, Mashanovich G, Gardes FY, Thomson DJ. Silicon optical modulators. Nat Photonics 2010; 4: 518-526.

41 Akkermans E, Montambeaux G. Mesoscopic Physics of Electrons and Photons. Cambridge: Cambridge University Press, 2007.

42 Levi L, Krivolapov Y, Fishman S, Segev M. Hyper-transport of light and stochastic acceleration by evolving disorder. Nat Phys 2012; 8: 912-917.

43 Scholak T, de Melo F, Wellens T, Mintert F, Buchleitner A. Efficient and coherent excitation transfer across disordered molecular networks. Phys Rev E 2011; 83: 021912.

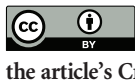

This work is licensed under a Creative Commons Attribution 3.0 Unported License. The images or other third party material in this article are included in the article's Creative Commons license, unless indicated otherwise in the credit line; if the material is not included under the Creative Commons license, users will need to obtain permission from the license holder to reproduce the material. To view a copy of this license, visit http://creativecommons.org/licenses/by/3.0/ 